QUARTERLY OF APPLIED MATHEMATICS

VOLUME LXIII, NUMBER 4

DECEMBER 2005, PAGES 681-690

S $0033-569 X(05) 00979-4$

Article electronically published on September 27, 2005

\title{
ON AN IMPROVED ELASTIC DISSIPATION MODEL FOR A CANTILEVERED BEAM
}

\author{
BY \\ M. A. ZARUBINSKAYA (Delft Institute of Applied Mathematics, Faculty of Electrical Engineering, \\ Mathematics and Computer Science, Delft University of Technology, Mekelweg 4, 2628 CD Delft, \\ The Netherlands) \\ AND \\ W. T. VAN HORSSEN (Delft Institute of Applied Mathematics, Faculty of Electrical Engineering, \\ Mathematics and Computer Science, Delft University of Technology, Mekelweg 4, 2628 CD Delft, \\ The Netherlands)
}

Abstract. In this paper we will study an improved elastic dissipation model for a cantilevered beam, where the damping is assumed to be proportional to the bending rate of the beam. For an earlier formulated dissipation model for the cantilevered beam it has been recently shown that damping will not always be generated. However, for the improved dissipation model it will be shown in this paper that damping will always be generated.

1. Introduction. For many years different approaches were used to describe energy dissipation in oscillating elastic bodies such as beams (see [1]-4]). However, many approaches (such as molecular theories) are too complicated to use in practice. As a result, different phenomenological models are used in mechanics. At the end of the nineteenth century, Kelvin and Voigt noted that damping rates tend to increase with frequency. At the end of the last century, Chen and Russell proposed the following dissipation model (see [4):

$$
\ddot{x}+B \dot{x}+A x=0,
$$

where $A$ is an elastic operator, and where $B$ is related in various ways to the positive square root, $A^{1 / 2}$, of $A$. For beam equations this approach was generalized and developed further by Russell in [1, 2]. Russell studied a new phenomenological dissipation model for a beam, where the damping is assumed to be proportional to the bending rate of the beam

$$
u_{t t}-\delta u_{t x x}+u_{x x x x}=0,
$$

Received February 2, 2005 and, in revised form, on March 16, 2005.

2000 Mathematics Subject Classification. Primary 35B05, 35Q72, 74H45.

E-mail address: maria@dv.twi.tudelft.nl

E-mail address: W.T.vanHorssen@ewi.tudelft.nl 


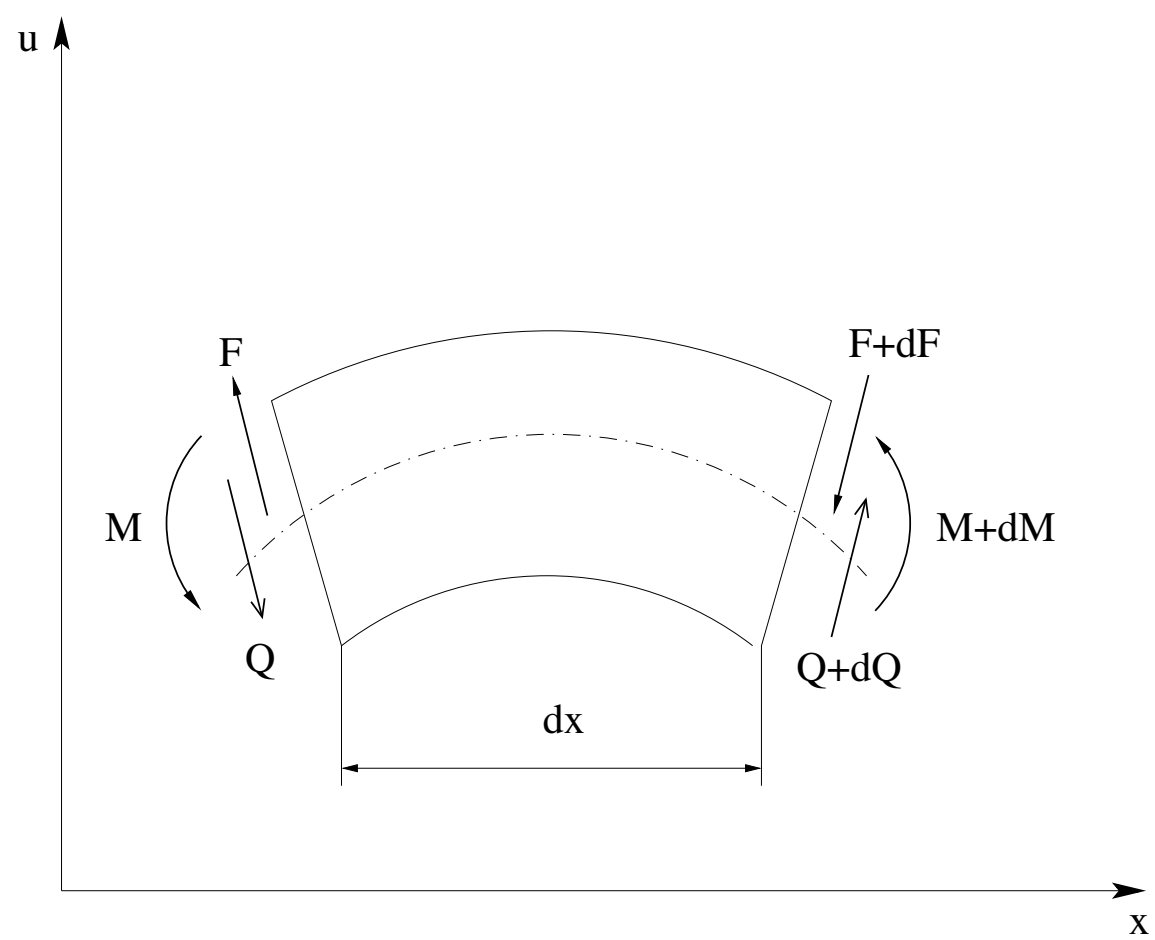

FIG. 1. Forces and moments acting on an element of the beam.

where $u(x, t)$ is the displacement of the beam in the vertical direction, and $\delta$ is a positive damping constant. No derivation of the dissipation term $\delta u_{t x x}$ is given in [1, [2]. However, it is noted that this new model has good mathematical properties. For example, in the case for simply supported beams it turns out that damping rates increase with frequency. Unfortunately for a cantilevered beam it turns out in [1] that $A$ and $B$ in (1.1) do not commute. Russell showed in $[3$ that it is, in general, not true that the energy decreases monotonically. In [5] it has been shown for the cantilevered beam problem as formulated in [1, 2] that the first (the lowest) vibration mode is unstable. So, for this mode there certainly is no energy dissipation.

In this paper an improved dissipation model will be studied for the cantilevered beam. It will be shown that dissipation really occurs in the system. In Section 2 of this paper it will be indicated how this dissipation model can be obtained. By using the recently developed, adapted version of the method of separation of variables (see [5], [6]), we will study the dissipation model in Section 3 of this paper. It will turn out that three cases have to be distinguished, that is, $\delta=2, \delta>2$, and $0<\delta<2$. These cases will be studied in Sections 4, 5, and 6, respectively. Finally, in Section 7 of this paper some conclusions will be drawn. 
2. Mathematical model. In [1-4] the following partial differential equation for $u=u(x, t)$ has been introduced:

$$
\begin{aligned}
& u_{t t}-\delta u_{t x x}+u_{x x x x}=0, \quad \Longleftrightarrow \\
& u_{t t}+\left(u_{x x x}-\delta u_{t x}\right)_{x}=0, \quad 0<x<\pi, t>0,
\end{aligned}
$$

where $\delta$ is a positive damping parameter. In this model equation (2.1) it is assumed that the damping is proportional to the bending rate of the beam. For a cantilevered beam, the following boundary conditions have been used in [1]-4]:

$$
u(0, t)=u_{x}(0, t)=u_{x x}(\pi, t)=u_{x x x}(\pi, t)=0 .
$$

However, by looking carefully at the PDE (2.1) it should be observed (see also Fig. 1) that the shear force $F$ (given by the term $u_{x x x}$ ) is in some sense related to the damping force $Q$ (given by the term $\delta u_{x t}$ ). So, the damping torque is assumed to be negatively proportional to the angular velocity. Now at the free end of the cantilevered beam the angular velocity is usually not zero. Hence, based on the structure of the PDE (2.1) the boundary condition $u_{x x x}(\pi, t)=0$ should be replaced by $u_{x x x}(\pi, t)-\delta u_{x t}(\pi, t)=0$. In this paper we propose the following boundary value problem for $u=u(x, t)$ as an improved elastic dissipation model for a cantilevered beam:

$$
\begin{aligned}
& u_{t t}-\delta u_{t x x}+u_{x x x x}=0, \quad 0<x<\pi, t>0, \\
& u(0, t)=u_{x}(0, t)=u_{x x}(\pi, t)=0, \quad t \geq 0, \\
& u_{x x x}(\pi, t)=\delta u_{x t}(\pi, t), \quad t \geq 0,
\end{aligned}
$$

where $\delta$ is a positive constant. For the boundary value problem (2.3) it can readily be shown that the energy $E(t)$, given by

$$
E(t)=\frac{1}{2} \int_{0}^{\pi}\left(u_{t}^{2}(x, t)+u_{x x}^{2}(x, t)\right) d x
$$

decreases for increasing times. In fact, by using the boundary conditions in (2.3) it can be shown simply that

$$
\frac{d E}{d t}=-\delta \int_{0}^{\pi} u_{t x}^{2} d x \leq 0
$$

3. Analysis of the dissipation model. A solution of the boundary value problem (2.3) can be constructed by using the method of separation of variables. Recently it has been shown in [6] that the method can be applied to a much larger class of problems than is generally assumed. Substituting a solution of the form $X(x) T(t)$ into the PDE and dividing the so-obtained equation by $X(x) T(t)$, we find

$$
\frac{\ddot{T}}{T}-\delta \frac{\dot{T}}{T} \frac{X^{\prime \prime}}{X}+\frac{X^{\prime \prime \prime \prime}}{X}=0
$$

where $^{\prime}=\frac{\partial(\ldots)}{\partial x}$ and ${ }^{\prime}=\frac{\partial(\ldots)}{\partial t}$. Generally it is assumed that (3.1) cannot be separated because of the mixed term $-\delta \frac{\dot{T}}{T} \frac{X^{\prime \prime}}{X}$. However, by simply differentiating (3.1) with respect 
to $x$ or $t$ (see also [6]) we can separate the variables. For instance, differentiation with respect to $x$ yields

$$
-\delta \frac{\dot{T}}{T} \frac{d}{d x}\left(\frac{X^{\prime \prime}}{X}\right)+\frac{d}{d x}\left(\frac{X^{\prime \prime \prime \prime}}{X}\right)=0
$$

which can easily be separated, yielding

$$
\frac{\dot{T}}{T}=\lambda
$$

where $\lambda$ is a complex valued separation constant. From (3.3) it follows that $\ddot{T}=\lambda \dot{T}=$ $\lambda^{2} T$, and then it can easily be deduced from (2.3) and (3.1) that $X(x)$ has to satisfy the following boundary value problem:

$$
\begin{gathered}
X^{\prime \prime \prime \prime}-\delta \lambda X^{\prime \prime}+\lambda^{2} X=0, \quad 0<x<\pi, \\
X(0)=X^{\prime}(0)=X^{\prime \prime}(\pi)=0, \quad X^{\prime \prime \prime}(\pi)=\delta \lambda X(\pi),
\end{gathered}
$$

where $\lambda=\lambda_{1}+i \lambda_{2}$ with $\lambda_{1}$ and $\lambda_{2} \in \mathbb{R}$. By considering the characteristic equation

$$
k^{4}-\delta \lambda k^{2}+\lambda^{2}=0 \quad \Longleftrightarrow \quad\left(k^{2}-\frac{\delta \lambda}{2}\right)^{2}+\frac{\lambda^{2}}{4}\left(4-\delta^{2}\right)=0
$$

for the differential equation (3.4), it is obvious that we have to consider three cases: $\delta=2, \delta>2$, and $0<\delta<2$. These three cases will be studied in the next three sections. It will be shown that nontrivial solutions for (3.4) can be found in all three cases. From (3.3) the time-dependent behaviour of a nontrivial solution $X(x) T(t)$ for (2.3) can be determined. It is obvious from (3.3) that arbitrary vibrations of the cantilevered beam can only be damped out if all eigenvalues $\lambda$ have a negative real part; that is, $\lambda_{1}$ should be negative for all vibration modes.

4. The case $\delta=2$. In this section we will study the boundary value problem (3.4)(3.5) with $\delta=2$. The characteristic equation for the differential equation (3.4) becomes in this case

$$
\left(k^{2}-\lambda\right)^{2}=0,
$$

where $\lambda=\lambda_{1}+i \lambda_{2}$ with $\lambda_{1}, \lambda_{2} \in \mathbb{R}$. It can be shown simply that, for $\lambda_{2}=0$, the boundary value problem (3.4) - (3.5) has only trivial solutions. For $\lambda_{2} \neq 0$ the characteristic equation (4.1) has as roots

$$
\zeta_{1}+i \zeta_{2}, \quad \text { and } \quad-\zeta_{1}-i \zeta_{2},
$$

where

$$
\zeta_{1}=\sqrt{\frac{\sqrt{\lambda_{1}^{2}+\lambda_{2}^{2}}+\lambda_{1}}{2}}, \quad \zeta_{2}=\sqrt{\frac{\sqrt{\lambda_{1}^{2}+\lambda_{2}^{2}}-\lambda_{1}}{2}} .
$$

Each root has multiplicity two. Putting $k=\zeta_{1}+i \zeta_{2}$, the general solution of the differential equation (3.4) can now be written as

$$
X(x)=C_{1} \cosh (k x)+C_{2} \sinh (k x)+C_{3} x \cosh (k x)+C_{4} x \sinh (k x),
$$

where $C_{1}, C_{2}, C_{3}$, and $C_{4}$ are complex valued constants of integration. By substituting (4.3) into the boundary conditions (3.5) we obtain a system of four linear, homogeneous 
TABLE 1. Approximations of the first ten eigenvalues $\lambda=\lambda_{1}+i \lambda_{2}$

for the case $\delta=2$.

\begin{tabular}{|c|c|r|}
\hline & \multicolumn{2}{|c|}{$\delta=2$} \\
\hline Nr. & $\lambda_{1}$ & \multicolumn{1}{|c|}{$\lambda_{2}$} \\
\hline 1 & -0.569648 & 0.568575 \\
2 & -3.196156 & 2.351548 \\
3 & -7.934745 & 4.418493 \\
4 & -14.729552 & 6.690023 \\
5 & -23.558855 & 9.116748 \\
6 & -34.411923 & 11.668342 \\
7 & -47.284223 & 14.324380 \\
8 & -62.166337 & 17.070178 \\
9 & -79.060929 & 19.894671 \\
10 & -97.964247 & 22.789222 \\
\hline
\end{tabular}

equations for $C_{1}, C_{2}, C_{3}$, and $C_{4}$. To have a nontrivial solution, the determinant of the coefficient matrix has to be zero, yielding

$$
-\left(k^{2}+2 \lambda\right) \cosh (k \pi)^{2}+\pi^{2} k^{4}-k^{2}\left(3+2 \lambda \pi^{2}\right)+2 \lambda=0 .
$$

Taking apart real and imaginary parts in (4.4), we get a system of two nonlinear equations for $\zeta_{1}$ and $\zeta_{2}$ (note that $k=\zeta_{1}+i \zeta_{2}$ with $\zeta_{1}$ and $\zeta_{2}$ given by (4.2)):

$$
\begin{aligned}
\frac{3}{2}\left(\zeta_{1}^{2}-\zeta_{2}^{2}\right) \cosh \left(2 \pi \zeta_{1}\right) \cos \left(2 \pi \zeta_{2}\right) & -3 \zeta_{1} \zeta_{2} \sinh \left(2 \pi \zeta_{1}\right) \sin \left(2 \pi \zeta_{2}\right)+\pi^{2}\left(\zeta_{1}^{4}+\zeta_{2}^{4}\right) \\
& +\frac{5}{2}\left(\zeta_{1}^{2}-\zeta_{2}^{2}\right)-6 \pi \zeta_{1}^{2} \zeta_{2}^{2}=0 \\
\frac{3}{2}\left(\zeta_{1}^{2}-\zeta_{2}^{2}\right) \sinh \left(2 \pi \zeta_{1}\right) \sin \left(2 \pi \zeta_{2}\right) & +3 \zeta_{1} \zeta_{2} \cosh \left(2 \pi \zeta_{1}\right) \cos \left(2 \pi \zeta_{2}\right)+4 \pi^{2} \zeta_{1} \zeta_{2}\left(\zeta_{1}^{2}-\zeta_{2}^{2}\right) \\
& +5 \zeta_{1} \zeta_{2}=0
\end{aligned}
$$

Using the formula manipulation package MAPLE, numerical approximations of the solution of (4.5) can easily be obtained. Using these approximations and (4.2), the eigenvalues $\lambda=\lambda_{1}+i \lambda_{2}$ can be approximated. The first ten approximations of the eigenvalues $\lambda$ of the boundary value problem (3.4)-(3.5) are listed in Table 1 .

5. The case $\delta>2$. In this section we will study the boundary value problem (3.4)(3.5) with $\delta>2$. The characteristic equation for the differential equation (3.4) is

$$
k^{4}-\lambda \delta k^{2}+\lambda^{2}=0,
$$

where $\lambda=\lambda_{1}+i \lambda_{2}$ with $\lambda_{1}, \lambda_{2} \in \mathbb{R}$. It is easy to show that, for $\lambda_{2}=0$, the boundary value problem (3.4)-3.5) has only trivial solutions. For $\lambda_{2} \neq 0$ it follows from the characteristic equation (5.1) that

$$
k^{2}=\lambda\left(\frac{\delta}{2}+\frac{1}{2} \sqrt{\delta^{2}-4}\right), \quad \text { or } \quad k^{2}=\frac{\lambda}{\left(\frac{\delta}{2}+\frac{1}{2} \sqrt{\delta^{2}-4}\right)} .
$$


Putting $a=\left(\frac{\delta}{2}+\frac{1}{2} \sqrt{\delta^{2}-4}\right)$ it follows from (5.2) that $k^{2}=\lambda a$ or $k^{2}=\frac{\lambda}{a}$. And so, the roots of the characteristic equation (5.1) are

$$
\text { ap, } \quad-a p, \quad p, \quad \text { and }-p,
$$

where $p=\xi_{1}+i \xi_{2}$ with

$$
\xi_{1}=\sqrt{\frac{\sqrt{\lambda_{1}^{2}+\lambda_{2}^{2}}+\lambda_{1}}{2}} \quad \text { and } \quad \xi_{2}=\sqrt{\frac{\sqrt{\lambda_{1}^{2}+\lambda_{2}^{2}}-\lambda_{1}}{2}} .
$$

For $\lambda_{2} \neq 0$, the solution of the differential equation (3.4) can now be written as

$$
X(x)=C_{1} \cosh (p x)+C_{2} \sinh (p x)+C_{3} \cosh (a p x)+C_{4} \sinh (a p x),
$$

where $C_{1}, C_{2}, C_{3}$, and $C_{4}$ are complex valued constants of integration. By substituting (5.4) into the boundary conditions in (3.5), we obtain a system of four linear, homogeneous equations for $C_{1}, C_{2}, C_{3}$, and $C_{4}$. To have a nontrivial solution the determinant of the coefficient matrix has to be zero, yielding

$$
\begin{aligned}
a\left(a^{2} p^{2}+p^{2}-2 \delta \lambda\right) \sinh (a p \pi) \sin (p \pi)+\left(a^{2} \delta \lambda\right. & \left.+\delta \lambda-2 a^{2} p^{2}\right) \cosh (a p \pi) \cos (p \pi) \\
& +a^{4} p^{2}-a^{2} \delta \lambda+p^{2}-\delta \lambda=0 .
\end{aligned}
$$

Taking apart the real and imaginary parts in (5.5), we finally obtain a system of two nonlinear equations for $\xi_{1}$ and $\xi_{2}$ (note that $p=\xi_{1}+i \xi_{2}$ with $\xi_{1}$ and $\xi_{2}$ given by (5.3)):

$$
\begin{aligned}
\frac{1}{2}(\delta & -a)(a+1)^{2}\left(\xi_{1}^{2}-\xi_{2}^{2}\right) \cosh \left((a-1) \pi \xi_{1}\right) \cos \left((a-1) \pi \xi_{2}\right) \\
& +\frac{1}{2}(\delta+a)(a-1)^{2}\left(\xi_{1}^{2}-\xi_{2}^{2}\right) \cosh \left((a+1) \pi \xi_{1}\right) \cos \left((a+1) \pi \xi_{2}\right) \\
& -(\delta-a)(a+1)^{2} \xi_{1} \xi_{2} \sinh \left((a-1) \pi \xi_{1}\right) \sin \left((a-1) \pi \xi_{2}\right) \\
& -(\delta+a)(a-1)^{2} \xi_{1} \xi_{2} \sinh \left((a+1) \pi \xi_{1}\right) \sin \left((a+1) \pi \xi_{2}\right) \\
& +\left(a^{4}-a^{2} \delta-\delta+1\right)\left(\xi_{1}^{2}-\xi_{2}^{2}\right) \\
= & 0 \\
\frac{1}{2}(\delta- & a)(a+1)^{2}\left(\xi_{1}^{2}-\xi_{2}^{2}\right) \sinh \left((a-1) \pi \xi_{1}\right) \sin \left((a-1) \pi \xi_{2}\right) \\
& +\frac{1}{2}(\delta+a)(a-1)^{2}\left(\xi_{1}^{2}-\xi_{2}^{2}\right) \sinh \left((a+1) \pi \xi_{1}\right) \sin \left((a+1) \pi \xi_{2}\right) \\
& +(\delta-a)(a+1)^{2} \xi_{1} \xi_{2} \cosh \left((a-1) \pi \xi_{1}\right) \cos \left((a-1) \pi \xi_{2}\right) \\
& +(\delta+a)(a-1)^{2} \xi_{1} \xi_{2} \cosh \left((a+1) \pi \xi_{1}\right) \cos \left((a+1) \pi \xi_{2}\right) \\
& +2\left(a^{4}-a^{2} \delta-\delta+1\right) \xi_{1} \xi_{2} \\
= & 0 .
\end{aligned}
$$

Numerical approximations of the solution of the previous system can easily be obtained by using the formula manipulation package MAPLE. The first ten approximations of the eigenvalues $\lambda$ are listed in Table 2 for $\delta=2.001, \delta=3$ and $\delta=10$. 
TABLE 2. Approximations of the first ten eigenvalues $\lambda=\lambda_{1}+i \lambda_{2}$

for the case $\delta>2$.

\begin{tabular}{|c|c|c|c|c|c|c|}
\hline & \multicolumn{2}{|c|}{$\delta=2.001$} & \multicolumn{2}{c|}{$\delta=3$} & \multicolumn{2}{c|}{$\delta=10$} \\
\hline Nr. & \multicolumn{1}{|c|}{$\lambda_{1}$} & \multicolumn{1}{c|}{$\lambda_{2}$} & $\lambda_{1}$ & $\lambda_{2}$ & $\lambda_{1}$ & $\lambda_{2}$ \\
\hline 1 & -0.559725 & 0.530103 & -0.649612 & 0.259728 & -0.202361 & 0.082725 \\
2 & -3.109869 & 2.249036 & -1.896037 & 0.482367 & -0.404255 & 0.115733 \\
3 & -7.705232 & 4.235023 & -3.766306 & 0.587929 & -0.673610 & 0.148573 \\
4 & -14.293713 & 6.414330 & -6.162337 & 0.846289 & -1.414891 & 0.214689 \\
5 & -22.854824 & 8.737879 & -9.234287 & 0.922084 & -1.886259 & 0.248083 \\
6 & -33.378299 & 11.174803 & -12.845145 & 1.255758 & -2.424643 & 0.281221 \\
7 & -45.858192 & 13.703768 & -21.970719 & 1.580228 & -3.030494 & 0.313897 \\
8 & -60.290729 & 16.308973 & -27.644080 & 1.730575 & -3.704214 & 0.346494 \\
9 & -76.673362 & 18.978011 & -33.566568 & 1.854684 & -4.445579 & 0.379587 \\
10 & -95.004283 & 21.701164 & -40.469762 & 2.193531 & -5.253938 & 0.413208 \\
\hline
\end{tabular}

6. The case $0<\delta<2$. In this section we will study the boundary value problem (3.4) - 3.5) with $0<\delta<2$. The characteristic equation for the differential equation (3.4) has the form (5.1). It can be shown elementarily that, for $\lambda_{2}=0$, the boundary value problem (3.4)-(3.5) has only trivial solutions. For $\lambda_{2} \neq 0$ it follows from the characteristic equation (5.1) that

$$
k^{2}=\lambda\left(\frac{\delta}{2}+i \frac{\sqrt{4-\delta^{2}}}{2}\right), \quad \text { or } \quad k^{2}=\frac{\lambda}{\frac{\delta}{2}+i \frac{\sqrt{4-\delta^{2}}}{2}} .
$$

Putting $a=\frac{\delta}{2}+i \frac{\sqrt{4-\delta^{2}}}{2}$ it follows from (6.1) that $k^{2}=\lambda a$, or $k^{2}=\frac{\lambda}{a}$. And so, the roots of the characteristic equation (5.1) are

$$
a p_{1}, \quad-a p_{1}, \quad p_{1}, \quad-p_{1},
$$

where $p_{1}=\eta_{1}+i \eta_{2}$ with

$$
\begin{aligned}
& \eta_{1}=\frac{1}{2}\left(\sqrt{\frac{\sqrt{\lambda_{1}^{2}+\lambda_{2}^{2}}+\lambda_{1}}{2}(2+\delta)}-\sqrt{\frac{\sqrt{\lambda_{1}^{2}+\lambda_{2}^{2}}-\lambda_{1}}{2}(2-\delta)}\right), \\
& \eta_{2}=\frac{1}{2}\left(\sqrt{\frac{\sqrt{\lambda_{1}^{2}+\lambda_{2}^{2}}-\lambda_{1}}{2}(2+\delta)}+\sqrt{\frac{\sqrt{\lambda_{1}^{2}+\lambda_{2}^{2}}+\lambda_{1}}{2}(2-\delta)}\right) .
\end{aligned}
$$

As in Section 5, the solution of the differential equation (3.4) can be written in the form (5.4). Again we obtain a system of four linear, homogeneous equations for $C_{1}, C_{2}, C_{3}$, and $C_{4}$ by substituting (5.4) into the boundary conditions (3.5). To have a nontrivial solution the determinant of the coefficient matrix has to be zero, yielding (5.5). The only difference now with the previous section is that $a$ and $p_{1}$ are both complex valued. Taking apart the real and imaginary parts in equation (5.5), we obtain a system of two 
nonlinear equations for $\lambda_{1}$ and $\lambda_{2}$ (note that $p_{1}=\eta_{1}+i \eta_{2}, a=a_{1}+i a_{2}=\frac{\delta}{2}+i \frac{\sqrt{4-\delta^{2}}}{2}$ ):

$$
\begin{aligned}
&\left(a_{1}^{5}-\right.\left.10 a_{1}^{3} a_{2}^{2}+5 a_{1} a_{2}^{4}+a_{1}-\delta\left(a_{1}^{2}-a_{2}^{2}+1\right)\right) \lambda_{1}+2 a_{1} a_{2}\left(-2 a_{1}^{3}+2 a_{1} a_{2}^{2}+\delta\right) \lambda_{2} \\
&+\frac{1}{2}\left[\left(a_{1}^{4}-6 a_{1}^{2} a_{2}^{2}+a_{2}^{4}+a_{1}^{2}-a_{2}^{2}-2 a_{1} \delta\right) \lambda_{1}+a_{2}\left(a_{1} a_{2}^{2}-3 a_{1}^{3}-a_{1}+2 \delta\right) \lambda_{2}\right] \\
& \cdot\left(\cosh \left(b_{1}\right) \cos \left(b_{2}\right)-\cosh \left(b_{3}\right) \cos \left(b_{4}\right)\right) \\
&-\frac{1}{2}\left[2 a_{2}\left(2 a_{1}^{3}-2 a_{1} a_{2}^{2}+a_{1}-\delta\right) \lambda_{1}+a_{1}\left(a_{1}^{3}-3 a_{1} a_{2}^{2}+a_{1}-2 \delta\right) \lambda_{2}\right] \\
& \cdot\left(\sinh \left(b_{1}\right) \sin \left(b_{2}\right)-\sinh \left(b_{3}\right) \sin \left(b_{4}\right)\right) \\
&+\frac{1}{2}\left[\left(-2 a_{1}^{3}+6 a_{1} a_{2}^{2}+\delta\left(a_{1}^{2}-a_{2}^{2}+1\right)\right) \lambda_{1}+2 a_{1} a_{2}\left(2 a_{1}-\delta\right) \lambda_{2}\right] \\
& \cdot\left(\cosh \left(b_{1}\right) \cos \left(b_{2}\right)+\cosh \left(b_{3}\right) \cos \left(b_{4}\right)\right) \\
&-\frac{1}{2}\left[2 a_{2}\left(a_{2}^{2}-3 a_{1}^{2}+a_{1} \delta\right) \lambda_{1}+\left(-2 a_{1}^{3}+2 a_{1} a_{2}^{2}+\delta\left(a_{1}^{2}-a_{2}^{2}+1\right)\right) \lambda_{2}\right] \\
& \cdot\left(\sinh \left(b_{1}\right) \sin \left(b_{2}\right)+\sinh \left(b_{3}\right) \sin \left(b_{4}\right)\right) \\
&= 0 \\
& a_{2}\left(5 a_{1}^{4}-10 a_{1}^{2} a_{2}^{2}+a_{2}^{4}+1-2 a_{1} \delta\right) \lambda_{1}+\left(a_{1}^{5}-6 a_{1}^{3} a_{2}^{2}+a_{1} a_{2}^{4}+a_{1}-\delta\left(a_{1}^{2}-a_{2}^{2}+1\right)\right) \lambda_{2} \\
&+\frac{1}{2}\left[\left(a_{1}^{4}-6 a_{1}^{2} a_{2}^{2}+a_{2}^{4}+a_{1}^{2}-a_{2}^{2}-2 a_{1} \delta\right) \lambda_{1}+a_{2}\left(a_{1} a_{2}^{2}-3 a_{1}^{3}-a_{1}+2 \delta\right) \lambda_{2}\right] \\
& \quad \cdot\left(\sinh \left(b_{1}\right) \sin \left(b_{2}\right)-\sinh \left(b_{3}\right) \sin \left(b_{4}\right)\right) \\
&+\frac{1}{2}\left[2 a_{2}\left(2 a_{1}^{3}-2 a_{1} a_{2}^{2}+a_{1}-\delta\right) \lambda_{1}+a_{1}\left(a_{1}^{3}-3 a_{1} a_{2}^{2}+a_{1}-2 \delta\right) \lambda_{2}\right] \\
& \cdot\left(\cosh \left(b_{1}\right) \cos \left(b_{2}\right)-\cosh \left(b_{3}\right) \cos \left(b_{4}\right)\right) \\
&+\frac{1}{2}\left[\left(-2 a_{1}^{3}+6 a_{1} a_{2}^{2}+\delta\left(a_{1}^{2}-a_{2}^{2}+1\right)\right) \lambda_{1}+2 a_{1} a_{2}\left(2 a_{1}-\delta\right) \lambda_{2}\right] \\
& \quad \cdot\left(\sinh \left(b_{1}\right) \sin \left(b_{2}\right)+\sinh \left(b_{3}\right) \sin \left(b_{4}\right)\right) \\
&+\frac{1}{2}\left[2 a_{2}\left(a_{2}^{2}-3 a_{1}^{2}+a_{1} \delta\right) \lambda_{1}+\left(-2 a_{1}^{3}+2 a_{1} a_{2}^{2}+\delta\left(a_{1}^{2}-a_{2}^{2}+1\right)\right) \lambda_{2}\right] \\
& \cdot\left(\cosh \left(b_{1}\right) \cos \left(b_{2}\right)+\cosh \left(b_{3}\right) \cos \left(b_{4}\right)\right) \\
&=0,
\end{aligned}
$$

where

$$
\begin{array}{ll}
b_{1}=\left(\left(a_{1}+1\right) \eta_{1}-a_{2} \eta_{2}\right) \pi, & b_{2}=\left(a_{2} \eta_{1}+\left(a_{1}+1\right) \eta_{2}\right) \pi \\
b_{3}=\left(\left(a_{1}-1\right) \eta_{1}-a_{2} \eta_{2}\right) \pi, & b_{4}=\left(a_{2} \eta_{1}+\left(a_{1}-1\right) \eta_{2}\right) \pi,
\end{array}
$$

and where $\eta_{1}$ and $\eta_{2}$ are given by (6.2). Using the formula manipulation package MAPLE, numerical approximations of the solution of the previous system can easily be obtained. The first ten approximations of the eigenvalues $\lambda$ of the boundary value problem (3.4)(3.5) are listed in Table 3 for $\delta=1.9999, \delta=1.0$, and $\delta=0.1$. 
TABLE 3. Approximations of the first ten eigenvalues $\lambda=\lambda_{1}+i \lambda_{2}$

for the case $0<\delta<2$

\begin{tabular}{|c|c|c|c|c|l|l|}
\hline & \multicolumn{2}{|c|}{$\delta=1.9999$} & \multicolumn{2}{c|}{$\delta=1.0$} & \multicolumn{2}{c|}{$\delta=0.1$} \\
\hline Nr. & \multicolumn{1}{|c|}{$\lambda_{1}$} & \multicolumn{1}{c|}{$\lambda_{2}$} & \multicolumn{1}{c|}{$\lambda_{1}$} & $\lambda_{2}$ & \multicolumn{1}{c|}{$\lambda_{1}$} & $\lambda_{2}$ \\
\hline 1 & -0.547494 & 0.579371 & -0.164894 & 0.005946 & -0.108821 & 0.526155 \\
2 & -3.135791 & 2.404405 & -1.240607 & 0.705894 & -0.351004 & 3.389543 \\
3 & -7.820001 & 4.574476 & -3.173444 & 3.149545 & -0.640398 & 8.52632 \\
4 & -14.559196 & 6.982240 & -6.078811 & 7.370027 & -1.03256 & 15.571784 \\
5 & -23.329186 & 9.586525 & -10.000888 & 13.333311 & -1.527404 & 24.586572 \\
6 & -34.119770 & 12.356921 & -14.929597 & 21.031815 & -2.123395 & 35.587779 \\
7 & -46.925065 & 15.273024 & -20.861508 & 30.463386 & -2.89967 & 48.581077 \\
8 & -61.741344 & 18.320226 & -27.795212 & 41.627437 & -3.616871 & 63.568843 \\
9 & -78.566087 & 21.487563 & -35.730008 & 54.523756 & -4.513981 & 80.552240 \\
10 & -97.397507 & 24.766517 & -44.665516 & 69.152246 & -5.511228 & 99.531906 \\
\hline
\end{tabular}

7. Conclusion. In this paper an improved version of a dissipation model for a cantilevered beam has been studied. The damping is assumed to be proportional to the bending rate of the beam. For the cantilevered beam the relationship between the damping rates and the frequencies has been obtained by using the recently developed, adapted version of the method of separation of variables (see [6]). It should be remarked that this relationship also can be obtained by applying the Laplace transform method to (2.3). The boundary value problem (3.4)-(3.5) then also is obtained. It has been shown that this phenomenological model for the cantilevered beam always generates damping, and this was the goal of the paper. Although it has not been shown, it seems most likely that the eigenfunctions form a Riesz basis for an appropriate energy space for the problems studied in this paper. It is well known that damping rates tend to increase with the frequency. Laboratory results as given in [3] for composite and wooden beams indicate a predominantly linear relationship between the damping rate and the frequency. For simply supported beams, it is obvious that the relationship between the damping rate and the frequency is linear. However, using this improved model for the cantilevered beam, our calculations indicate that there is not a strong linear dependence between the damping rate and the frequency.

\section{REFERENCES}

[1] D.L. Russell, On the positive root of the fourth derivative operator, Quarterly of Applied Mathematics, 16, No. 4, (1988), pp. 751-773. MR0973388 (90f:47069)

[2] D.L. Russell, A comparison of certain elastic dissipation mechanisms via decoupling and projection techniques, Quarterly of Applied Mathematics, 19, No. 2, (1991), pp. 373-396. MR.1106398 (92e:73029)

[3] D.L. Russell, On mathematical models for the elastic beam with frequency-proportional damping, Chapter 4 in Control and Estimation in Distributed Parameter Systems, edited by H.T. Banks, 11 of Frontiers in Applied Mathematics, SIAM Pubs., (1992).

[4] G. Chen, D.L. Russell, A mathematical model for linear elastic systems with structural damping, Quarterly of Applied Mathematics, 16, No. 1, (1982), pp. 433-454. MR0644099 (83f:70034) 
[5] W.T. van Horssen, M.A. Zarubinskaya, On an elastic dissipation model for a cantilevered beam, Quarterly of Applied Mathematics, 61, No. 3, (2002), pp.565-573. MR1999837 (2004d:74041)

[6] W.T. van Horssen, On the applicability of the method of separation of variables for partial difference equations, Journal of Difference Equations and Applications, 8, No. 1, (2002), pp. 53-60. MR1884591 (2003d:39024) 